\title{
NIVEL DE DESARROLLO DEL VALOR RESPONSABILIDAD EN ALUMNOS DE LAS ESCUELAS PROFESIONALES DE EDUCACIÓN Y PSICOLOGÍA DE LA UNIVERSIDAD PRIVADA SAN PEDRO, HUARÁZ - ANCASH
}

\author{
LEVEL OF DEVELOPMENT OF THE VALUE RESPONSIBILITY IN STUDENTS \\ OF THE PROFESSIONAL SCHOOLS OF EDUCATION AND PSYCHOLOGY OF \\ THE PRIVATE UNIVERSITY SAN PEDRO, HUARÁZ - ANCASH \\ Víctor Manuel Zegarra Chauca ${ }^{1}$, Jesús Manuel More López ${ }^{1}$, Félix Valerio Haro ${ }^{1}$, \\ José Antonio Legua Cárdenas ${ }^{2}$, Yasmín Jesús Vélez Chang ${ }^{2}$, Antonio Salomón Valderrama Romero ${ }^{3}$
}

\section{RESUMEN}

Objetivo: Conocer el nivel de desarrollo del valor responsabilidad en estudiantes de dos escuelas profesionales de la Universidad San Pedro. Material y Métodos: Se trató de una investigación descriptiva, con diseño no experimental de tipo transeccional; para lo cual se determinó una muestra de 97 alumnos mediante la técnica aleatoria simple; a quienes se aplicó los instrumentos cuestionario y guía de observación elaborados por los autores en función al marco teórico y tomando en cuenta el diseño de clasificación de indicadores de González et al (2001). Para el análisis e interpretación de resultados se hizo uso de la estadística descriptiva y de los métodos: análisis, síntesis y comparación. Resultados: se evidenciaron leves mejoras en los niveles medio y alto del valor responsabilidad en alumnos del IV ciclo respecto a los del I ciclo $(1.13 \%)$; igualmente mayor tendencia en el nivel alto de desarrollo del valor responsabilidad en alumnos que se dedican sólo a estudiar en comparación a aquellos que estudian y trabajan. Conclusión: en los estudiantes de los tres ciclos académicos de las dos escuelas profesionales de la Universidad existe prevalencia de la tendencia medianamente responsable de los alumnos (49.5\%)

Palabras clave: Valor moral; Responsabilidad; Actitud; Crisis de valores.

\section{ABSTRACT}

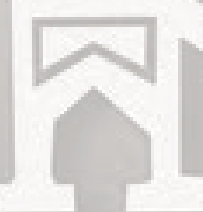

Objetive: To meet the developmental level of the student's accountability value of two professional schools of the University of San Pedro. Materials and Methods: This was a descriptive, nonexperimental design of transactional type, which was determined for a sample of 97 students by simple random technique, who completed the questionnaire instruments and observation guide developed by the authors according to the framework theoretical and design taking into account the classification of indicators Gonzalez et al (2001). For the analysis and interpretation of results was done using descriptive statistics and methods: analysis, synthesis and comparison. Results: showed slight improvements in medium and high levels of responsibility in students value the fourth cycle compared to the first cycle $(1.13 \%)$ also more likely in high value development responsibility in students that are dedicated only to study compared to those who study and work. Conclusion: the students of the three academic cycles of the two professional schools of the University is the prevalence of moderately trend responsible for the students $(49.5 \%)$

Key words: Moral value; Responsibility; Attitude; Values crisis.

Facultad de Ciencias Sociales, Educación y Comunicación, Universidad Nacional Santiago Antúnez de Mayolo. E-mail: mamore_08_31@hotmail.com 


\section{INTRODUCCIÓN}

Cada vez más, el nuevo orden que se impone en la sociedad requiere de profesionales más competentes. Este nivel de competitividad tiene que ver no solo con los conocimientos y habilidades adquiridas sino, también, con intereses, actitudes y valores a ser priorizados en docentes, trabajadores y estudiantes en formación.Pero para priorizar la práctica de valores en la vida del ser humano, en el sentido de que regulan su actuación como tal, no basta enseñar un conjunto de valores sino, más bien, hay que posibilitar su valoración. Es decir, que cada alumno o alumna debe reflexionar y discernir acerca de aquellos valores que desea hacer propios. Si bien vivimos en la era de la tecnología y del desarrollo extensivo de las telecomunicaciones ello no basta para decir que los valores han perdido preponderancia, si bien algunos no se expresan como antaño otros, en cambio, han aparecido por el dinamismo específico de la sociedad. En el caso del Perú, en la última década, no es común hablar de práctica de valores sino más bien de crisis de valores. Ello se evidencia elevados índices de corrupción, ascenso de la criminalidad, violencia juvenil, drogadicción, narcotráfico, protestas violentas, impunidad, etc.Esta crisis es la que está incidiendo en demasía en la dinámica familiar a tal punto que resquebrajan su unidad y no propician un espacio para el desarrollo natural de la razón respecto a ciertos valores mínimos. Posibilita que gran parte de los jóvenes de hoy muestren desinterés por la institución familiar al no cumplir una de sus funciones: el ser un ambiente donde la persona pueda expresarse y desarrollarse a plenitud, considere pertinente otro tipo de organización fuera del ambiente familiar (la calle). Este tipo de hechos ocasiona la declinación de valores fundamentales como la honestidad, tolerancia, responsabilidad y da origen a conductas reprochables en los jóvenes, principalmente, de los niveles básicos de ễucación. Las instituciones de educación superior no están ajenas a este tipo de situaciones. Se puede percibir comportamientos y actitudes poco responsables en los estudiantes como, por ejemplo: no asistencia a clases regularmente y lo hacen cuando estiman conveniente, presentan sus trabajos a última hora, realizan escasa actividad investigativa, incumplen promesas, entre otros.

Una adecuada educación en valores es vital para dinamizar las competencias ético - morales acorde a las nuevas circunstancias en las que vivimos tanto en los niveles básicos como superiores de educación. Este último nivel, a decir de Arana y Batista (2000), debe tributar a la sociedad en la formación de hombres que sean capaces de identificar, asimilar, optimizar, adaptar, mejorar y desarrollar tecnologías apropiadas que brinden soluciones adecuadas en cada instante; formación que posibilite cambiar la calificación técnica, social y humana.Esto significa que la universidad no debe ser sólo portadora de valores cognoscitivos o de tipo comunicativo sino de valores morales como el respeto, la tolerancia, la voluntad, la autonomía y la responsabilidad.

De la literatura revisada merece destacar diversos estudios acerca de los valores y su desarrollo en estudiantes: Entre ellos a los de Aldea (2001), Molina (2002), Gonzáles et al (2001); todos realizados en el extranjero. En el Perú, de Uribe (2002) y Mora (2003).

Tomando como referencia estos hechos nos formulamos la siguiente interrogante: ¿Cómo se manifiesta el nivel de desarrollo del valor responsabilidad en alumnos de las escuelas profesionales de Educación y Psicología de la Universidad San Pedro sede Huaraz? Como objetivo general de la investigación: determinar el nivel de desarrollo del valor responsabilidad en alumnos en las escuelas profesionales de Educación y Psicología de la Universidad "San Pedro" sede Huaraz.

La importancia de los resultados obtenidos y su aporte práctico está dada por la utilidad que tiene el diagnóstico que revela la información pertinente; el cual, permitirá elaborar y poner en práctica estrategias orientadas a mejorar la actitud responsable de los alumnos. Es decir, se justifican las acciones que se pretenden realizar con el fin de posibilitar que los jóvenes tomen conciencia acerca de la práctica de valores y modelen sus actitudes en bien del ejercicio profesional.

Conocer la magnitud de la problemática desde ámbitos cognoscitivos y reales en mayor medida, es lo que ha propiciado la realización del presente trabajo. Porque consideramos que existe una realidad y un nivel de exigencia no resuelta por métodos empíricos y que merece ser investigada. De hecho, puede orientarse su práctica desde el ámbito educativo. Nos queda como docentes incentivar esta práctica porque coadyuvará a formar jóvenes dedicados a afrontar con decisión los retos del futuro.

\section{MATERIALES Y MÉTODOS}

La investigación fue descriptiva, con diseño no experimental de tipo transeccional y justificada en la caracterización de los hechos y situaciones del objeto de investigación.

La población estuvo constituida por la totalidad de alumnos de las escuelas profesionales de Educación 
y Psicología. Educación en número de 124 y Psicología, 85 que suman en total 209. Distribuidos en 05 ciclos académicos. Todos con las mismas probabilidades de ser seleccionados.

La muestra estuvo conformada por la totalidad de los alumnos de los ciclos I y IV de Psicología en número de 40 y; I, II y IV de Educación, en número de 57; que asistieron a clases los días de aplicación de los instrumentos. En total 97. La técnica empleada para la selección de la muestra fue la aleatoria simple.

Para la recolección de datos se hizo uso de las técnicas de la encuesta con su instrumento el cuestionario y la observación con su instrumento: guía de observación.

Ambos instrumentos fueron elaborados por $10 \mathrm{~s}$ investigadores en función al marco teórico y teniendo en cuenta el diseño de clasificación de los indicadores de la variable en estudio planteado por González, et al. (2001).

Antes de la aplicación del cuestionario se realizó un pilotaje, con estudiantes de enfermería con el fin de precisar la estructuración de la técnica. Este pilotaje demostró que si se quería obtener datos que reflejaran la real dimensión de la problemática no bastaría la aplicación de un solo instrumento. Por tanto, se eligió dos con el fin de darle mayor consistencia a los datos.

Para cada una de las respuestas se empleó una escala valorativa de alto, medio y bajo. Después de seleccionada la muestra se aplicaron los instrumentos: primero la guía de observación y luego el cuestionario en los periodos establecidos.

Una vez recolectado los datos sobre conducta real manifiesta y opiniones de los alumnos con respecto al nivel de desarrollo del valor responsabilidad, se procedió a tabularlos, para luego analizarlos, interpretarlos y los resultados expresarlos en porcentajes; para, finalmente, arribar a conclusiones y expresar las recomendaciones pertinentes. Para ello se hizo uso de la estadística descriptiva.

\section{RESULTADOS Y DISCUSIÓN}

En los datos que se muestran a continuación precisan la forma como se percibe, en los alumnos la manifestación del valor responsabilidad en su vida universitaria; la cual ayudará a tomar conciencia y adoptar decisiones pertinentes en bien del ejercicio profesional.
Tabla 1. Consolidado de respuestas sobre nivel de desarrollo del valor responsabilidad en alumnos de las escuelas profesionales de Educación y Psicología

\begin{tabular}{|c|c|c|c|c|c|c|c|c|}
\hline & \multicolumn{2}{|c|}{ Ciclo I } & \multicolumn{4}{|c|}{ Ciclo II Ciclo IV } & \multirow[b]{2}{*}{$\mathbf{N}^{\circ}$} & \multirow[b]{2}{*}{$\%$} \\
\hline Valor & $\mathbf{N}^{\circ}$ & $\%$ & $\mathbf{N}^{\circ}$ & $\%$ & $\mathbf{N}^{\circ}$ & $\%$ & & \\
\hline Alto & 8 & 24 & 7 & 27 & 10 & 26 & 25 & 26 \\
\hline Med & 15 & 46 & 14 & 54 & 19 & 50 & 48 & 50 \\
\hline Bajo & 10 & 30 & 5 & 19 & 9 & 24 & 24 & 25 \\
\hline TOTAL & & 33 & & 66 & & 38 & 97 & 100 \\
\hline
\end{tabular}

Los resultados muestran una mayor tendencia en el nivel medio del valor responsabilidad tanto en alumnos de la Escuela de Educación como de Psicología. Tomando como referencia las actuales condiciones en las que vivimos en donde la práctica de determinados valores morales está venida a menos no se espera que la valoración alta o primera sea la de mayor ponderación o que tampoco tenga primacía la de bajo nivel. Lo ideal para no caer en crisis es vivirlos, con ello configuraríamos un mundo más humano. Este planteamiento se relaciona con lo señalado por Padilla (1996), Pereira (2004), Yarce (2004).

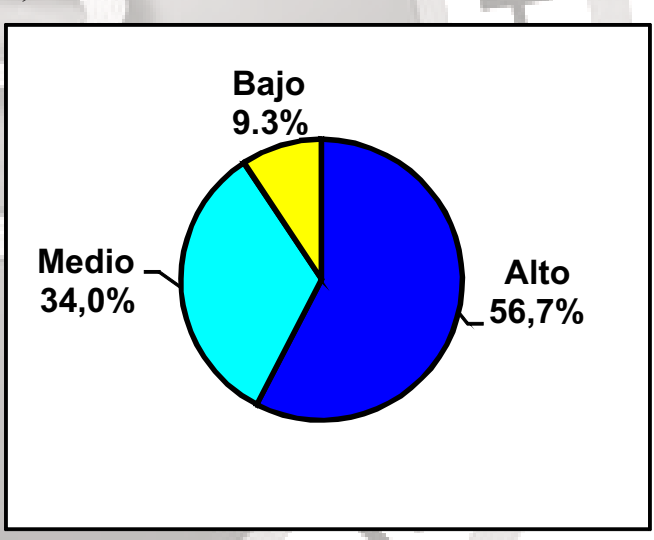

Figura 1. Definición del término

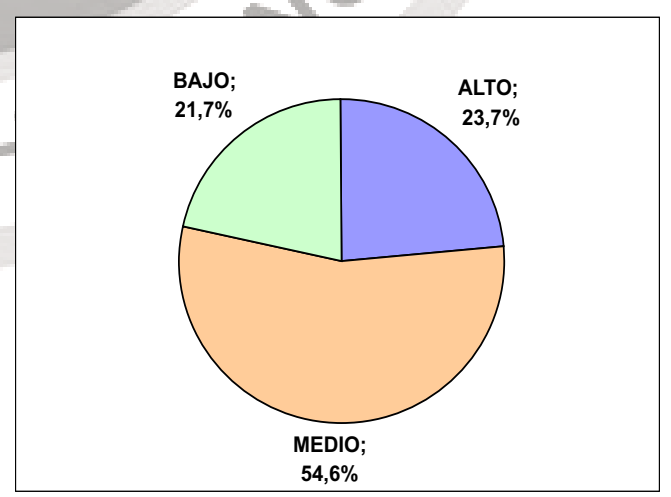

Figura 2. Conducta real manifiesta. Responsabilidad.

A través de los resultados podemos percibir que si bien los alumnos definen el valor responsabilidad de manera concreta tomando en cuenta los atributos que lo incluyen, en comparación a aquellos que 
solamente reconocen algunos de ellos o no lo precisan adecuadamente; esta tendencia no se refleja en la conducta real manifiesta en donde el actuar de manera responsable no refleja el mayor índice; sino, más bien, la segunda opción. Es decir, falta asumir el compromiso y la decisión para por lo menos acercarse a la mitad con el cumplimiento de los atributos considerados apropiados que, permita establecer un paralelismo entre lo que se sabe y lo que se hace sin marcadas diferencias, tal como se percibe en las figuras 1 y 2 .

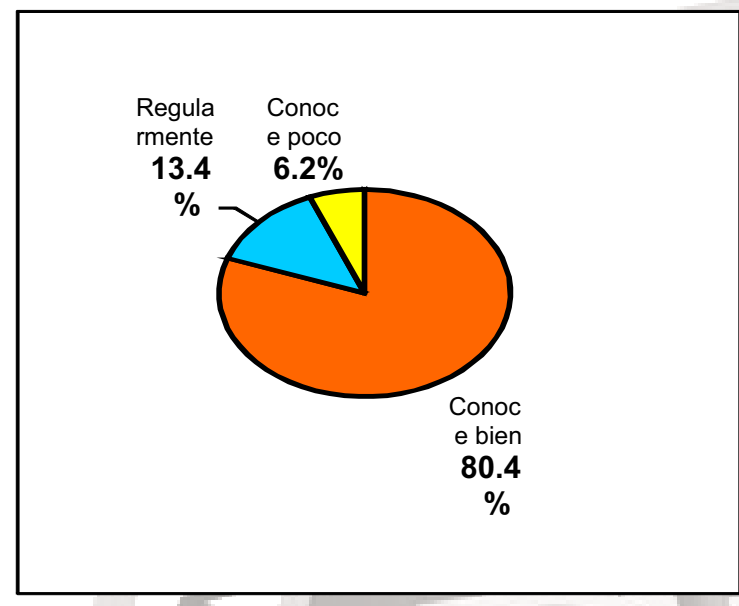

Figura 3. Atributos que definen al estudiante responsable

El estudiante precisa bien los atributos que definen ser responsable; está relacionado con cumplir con los trabajos y tareas de la mejor forma, que requiere de esfuerzo, vencer obstáculos y asumir las consecuencias de los actos; prima la necesidad interna antes que la externa. Pero quienes lo asumen como tal no llegan al $50 \%$. Más aún si logran comprender que el actuar de manera responsable no está supeditado a la obligación y presión; pero que algunas veces no debemos responder solo por nuestros actos sino también por los demás.



Figura 4. Motivos que inducen a actuar de manera responsable.
Cada ser humano siente la necesidad interna de actuar responsablemente sin que en ella incidan otros factores, mayormente, externos; Figura 4. Esto se refleja en el sentido en que somos nosotros quienes elegimos y hacemos valoraciones sobre la práctica de un determinado valor en función del grado de significación, regulación y orientación de la actividad humana Rubal, (2000); Carreras et al (2003).

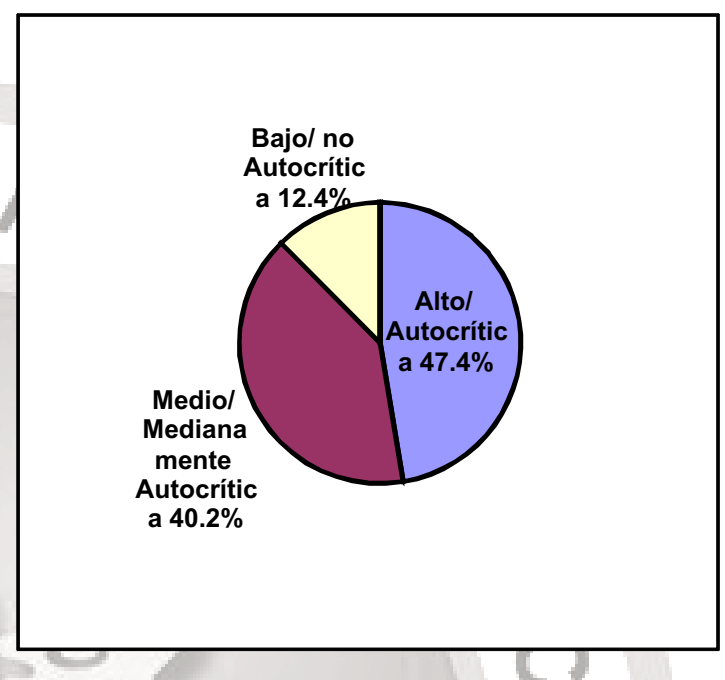

Figura 5. Nivel de autocrítica demostrada

Cuando se acepta asumir la responsabilidad de realizar un trabajo en grupo se debe ser capaz de responder no sólo por sus actos sino, también por lo de los demás; pero una vez concluido la actividad ser capaz de hacerse una autocrítica precisando los aspectos buenos y malos. Tal como se precisa en la figura 5, la tendencia no es tan marcada entre el nivel alto y medio de valoración autocrítica. Lo cual significa que en algunos casos es capaz de analizar sus conductas y reconocer sus limitaciones; en otros casos reconocer sólo algunos comportamientos y mostrarse más bien tolerante y justificativo. ¿cuál es la razón? Es una inquietud que queda por determinar y resolver asi como el porque determinados comportamientos y actitudes no se manifiestan en igualdad de condiciones como se conceptúan.

\section{CONCLUSIONES}

1. Al determinar el nivel de desarrollo del valor responsabilidad en los alumnos de los tres ciclos académicos de las escuelas de Educación y Psicología los resultados nos demuestran el predominio de la tendencia medianamente responsable (49.5), existiendo en alumnos del primer ciclo un gran porcentaje de la tendencia poco responsable o irresponsable.

2. Los resultados evidenciaron que existen 
leves mejoras en los niveles medio y alto del valor responsabilidad en alumnos del IV ciclo respecto a los de los primeros ciclos (1.13\%).

3.Los resultados demostraron mayor tendencia en el nivel alto de desarrollo del valor responsabilidad de los alumnos que se dedican a estudiar en comparación a aquéllos que estudian y trabajan $(12.5 \%)$. De igual forma, se observa que los alumnos que estudian y trabajan demuestran mayor tendencia en el nivel bajo del valor responsabilidad en un porcentaje superior al $7 \%$.

\section{REFERENCIA BIBLIOGRÁFICA}

Aldea, E. (2001) Estrategias educativas para trabajar en valores. Curso de perfeccionamiento dirigido a profesores de secundaria del sistema de educación municipal. Chillán. En http://www.campus-oei.org/valores/expert.html

Arana, My Batista, N. 2000. La educación en valores: Una propuesta pedagógica para la formación profesional. La Habana: ISPJAE.

Carreras, L; Eijó, P; Estany, A; Gomez, M; Guich, R; Mir, V; Ojeda, F; Planas, T; Serrats, M. 2003. Cómo educar en valores: Materiales, textos, recursos y técnicas. 12 ava. Edición. Madrid: Narcea.

Gonzalez, V; Kraftchenko, O y Ojalvo, V. 2001. Diagnóstico psicopedagógico de los valores. En Ojalvo, V. et al. La educación en valores en el contexto universitario. La Habana: Félix Valera.

Molina, T. 2002. Estrategia de evaluación del aprendizaje para la contribución al desarrollo de la responsabilidad profesional. Tesis de doctorado. La Habana: ISPJAE.
Mora, A. 2003. Gestión para la educación en valores. Tesis de doctorado. Chimbote: Universidad PrivadaSan Pedro.

Padilla, A. 1996. Educación en valores y su sentido. Revista pensamiento educativo. Educación de valores. Vol. 18. Facultad de Educación. Pontificia Universidad Católica de Chile.

Pereira, M. 2004. Educación en valores. Metodología e innovación educativa. México: Trillas.

Rubal, X. 2000. Los contenidos actitudinales: Anotaciones conceptuales y evaluativos. En Trillo, F. (Coord.) La educación en actitudes y valores: Dilemas para su enseñanza y evaluación. Homo Santa Fe: Homo Sapiens.

Uribe, C. 2002. Una experiencia de formación docente en cultura de paz. Lima: Pontificia Universidad Católica del Perú.

Yarce, J. 2004. Valor para educar en valores. Lima: Norma.

\section{HUACHO}

